\title{
Origin and Evolution
}

of Planetary and Satellite Systems 



\section{Origin and Evolution of Planetary and Satellite Systems}

Edited by

Diedrich Möhlmann and Heinz Stiller

With 61 Figures and 39 Tables

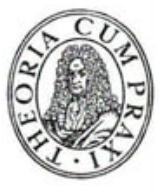

Akademie-Verlag Berlin 1989 
Authors:

Siegfried Franck, Zentralinstitut für Physik der Erde der AdW der DDR, Potsdam

Rudolf Gottfried, Dresden

Diedrich Möhlmann, Institut für Kosmosforschung der AdW der DDR, Berlin

Ingo Orgzall, Zentralinstitut für Physik der Erde der AdW der DDR, Potsdam

Ulrich Schmit, Institut für Kosmosforschung der AdW der DDR, Berlin

Frank Spahn, Institut für Kosmosforschung der AdW der DDR, Berlin

Hanno Sponholz, Institut für Kosmosforschung der AdW der DDR, Berlin Heinz Stiller, Akademie der Wissenschaften der DDR, Berlin

Richard Wäsch, Institut für Kosmosforschung der AdW der DDR, Berlin

ISBN 3-05-500278-4

Erschienen im Akademie-Verlag Berlin, Leipziger Straße 3-4, Berlin, DDR - 1086 (C) Akademie-Verlag Berlin 1989 Lizenznummer: $202 \cdot 100 / 441 / 88$ Printed in the German Democratic Republic Gesamtherstellung: VEB Druckhaus „Maxim Gorki", Altenburg, 7400 Lektor: Dipl.-Met. Heide Deutscher LSV 1495

Bestellnummer: 7636735 (9050) 05200 